\title{
Student attitudes to periodontology
}

A recent BDA survey asked readers of the BDA magazine Launchpad a number of questions about themselves and their opinions on the publication itself. Launchpad is sent to all students and Vocational Dental Practitioners (VDPs) that are also BDA members, and as such it represents the views and attitudes of those people literally just beginning their dental careers.

The survey was included with an issue of the magazine sent out to 3462 readers and only 438 (19\%) replied. This was a much lower response rate than the $66 \%$ achieved in the last survey in 1999, but at that time two reminders were sent out to readers as well as the questionnaire. Thus, the findings must be treated with caution as they are more likely to represent the views of those who are particularly interested in Launchpad. Despite this, 19\% is a very healthy response compared with most journal and magazine readership surveys. The good news is that the responses were pretty evenly spread out over all the dental schools around the UK.

The findings are very encouraging (albeit potentially biased as explained above). Ninety four per cent of responders claimed they read Launchpad, and virtually the same percentage liked the new design, liked the articles and trusted the content. Interestingly, virtually all the respondents were dental students, not VDPs, but the reason for this is not clear. Perhaps not surprisingly the only other publications the students claimed to read besides Launchpad were BDA News, the BDJ and Dental Update. The other unsurprising finding was that 93\% had internet access and e-mail.

However, one of the findings that was of interest was the question asking which areas of dentistry the readers were interested in, allowing the readers to include as many subjects as they wished. The most popular topic was oral surgery (62\%) with orthodontics following closely behind (53\%). Sadly (from my point of view) periodontology was virtually bottom of the list (29\%). It seems that, despite all the efforts in previous years to move dentistry away from a mechanical repair approach to a preventive oral health approach, the opinion of dental students (judged only by this survey) still tends towards the former. I not only find this inexplicable; I find it worrying.

Perhaps I should not be concerned after all, for the findings are only a reflection of interests at the initial stage of understanding - as the respondents were still dental students. Yet I would have thought that periodontology is often more interesting when one is dealing with the theoretical concepts rather than the pragmatic realities of trying to make it work in the real world; this survey suggests this is not so.

Of course, simply because people state that they are less interested in a topic does not mean they will give it less emphasis in their practice and in their individual treatment plans for patients - but one does begin to wonder. Our behaviour tends to follow our particular interests and values, and we are very good at rationalising our view of the world to make our actions fit into our particular proclivities. That is one reason why a patient can visit a number of different dentists and receive different treatment options, all genuinely based on what each individual feels is best. If dental students are more interested in every other aspect of clinical care than periodontology, does that mean their treatment options are less likely to have a sound periodontal base? It is more comfortable to hope not, but is it more realistic?

One possible reason for this apparent lack of interest in periodontolgy in comparison with the other clinical areas is the fact that most periodontal care is delegated to hygienists and therapists, who carry out the basic scaling and plaque control activities. The dentist's role is more of a directorial one, as befits the leader of the team. This would again suggest that dental students themselves still perceive their role as 'doers' rather than 'leaders', a worrying fact for those who believe the future of dentistry is a team approach with the dentist at the head.

Hmmm.

Mike Grace, Editor

m.grace@bda.org

doi: 10.1038/sj.bdj.4811519 